\title{
Função visual de crianças pseudofácicas por catarata infantil
}

\section{Visual Function in pseuphakic children with Pediatric cataract}

Patrícia da Rocha Pitta Ferraz', Debora Mayumi Sugano², Celso Lopez Fernandes ${ }^{3}$

\section{Resumo}

Objetivo: $\mathrm{O}$ tipo de ambliopia mais grave é o de privação, deste a causa mais comum é a catarata congênita. $\mathrm{O}$ objetivo deste estudo foi avaliar a função visual binocular de crianças operadas de julho de 2006 a junho de 2008 por cataratas da infância (congênita ou infantil). Métodos:Todas as crianças possuíam idade acima de 4 anos, operadas em idade tardia. Foram avaliadas 6 crianças e realizados testes de acuidade visual, motilidade ocular extrínseca, testes de Titmus e Luzes de Worth (perto e longe). Resultados: A média da idade do diagnóstico da catarata foi de 5,58 anos e o da primeira cirurgia de 8,83 anos. Cinquenta e $67 \%$ das crianças alcançaram acuidade visual pósoperatória maior que $0,4 \mathrm{em}$ olhos direito e esquerdo, respectivamente. Apenas uma criança não apresentou estereopsia e fusão aos testes de função sensorial. Todas as demais apresentaram fusão às luzes de Worth. No teste de Titmus, $2(33,3 \%)$ apresentaram estereopsia grosseira e $3(50 \%)$ boa ou ótima. Houve diferença estatísticamente significante $(\mathrm{p}=0,043)$ entre as acuidades visuais pré e pós- operatórias entre os olhos. Conclusão: Assim, neste estudo as crianças portadoras de catarata congênita ou infantil precoce de desenvolvimento lento e de catarata infantil tardia, apresentaram bom prognóstico visual após cirurgia com implante de lente intraocular no mesmo tempo cirúrgico, mesmo em idade avançada.

Descritores: Catarata/ congênito; Percepção de profundidade; Acuidade visual

${ }^{1}$ Residente do $3^{\circ}$ ano de Oftalmologia da Faculdade de Medicina do ABC - FMABC - Santo André (SP), Brasil.

${ }^{2}$ Professora colaboradora do setor de Estrabismo da Faculdade de Medicina do ABC - FMABC - Santo André (SP), Brasil.

${ }^{3}$ Chefe do setor de Estrabismo da Faculdade de Medicina do ABC - FMABC - Santo André (SP), Brasil.

Setor de Estrabismo da Disciplina de Oftalmologia da Faculdade de Medicina do ABC - FMABC - Santo André (SP), Brasil.

Recebido para publicação em: 17/7/2009 - Aceito para publicação em 1/10/2009 


\section{INTRODUÇÃO}

B inocularidade é o termo que se aplica à capacidade de aprender estímulos visuais com os dois olhos. Sua avaliação em um indivíduo divide-se em três esferas: a percepção macular simultânea, a fusão e a estereopsia. ${ }^{1}$ Cada uma destas esferas podem ser avaliadas separadamente, com testes específicos, e o conjunto de seus resultados pode graduar a binocularidade do indivíduo avaliado ${ }^{1}$.

A ausência ou diminuição da visão de um ou ambos os olhos sem alteração anatômica, chama-se ambliopia. Ocorre por interação binocular anormal (desvios oculares presentes ou não), privação de forma ou do estímulo visual. A importância da ambliopia consiste no fato de que as perdas visuais que a acompanham tornam-se irreversíveis se não precocemente tratadas. Os tipos de ambliopia mais graves são os que se desenvolvem por privação de estímulo ou desuso, das quais a causa mais comum é a catarata congênita ${ }^{1,2}$.

Cataratas são a maior causa de cegueira da infância, com prevalência de 1,2 a 6,0 casos por 10.000 nascimentos $^{3}$. O implante de lente intra-ocular para a correção da afacia em crianças, está sendo cada vez mais preconizado e aceito pelos especialistas da área, porém, continua controverso já que os resultados a longo prazo não são conhecidos ${ }^{4,5}$.

A cirurgia de catarata congênita ou do desenvolvimento é indicada em crianças com opacidade cristaliniana de $3 \mathrm{~mm}$ ou maior que acometa o eixo visual $^{6,7}$. O resultado visual de olhos operados de cataratas da infância depende da idade de início da catarata, da idade em que a cirurgia foi realizada, do tipo morfológico da catarata e o acompanhamento e tratamento da ambliopia ${ }^{7,8}$. Conforme a literatura sobre catarata congênita e do desenvolvimento, considera-se sucesso no tratamento quando a acuidade visual final obtida é igual ou maior que 20/40(logmar $)^{6,8}$.

Recentes estudos mostram bons resultados visuais no acompanhamento de crianças submetidas à cirurgia de catarata, porém, os resultados de função binocular destas crianças ainda são limitados ${ }^{3,6}$.

\section{Овjetivo}

O objetivo deste estudo foi estudar a função visual binocular de crianças portadoras de cataratas da infância (congênita ou infantil), do setor de Oftalmopediatria da Disciplina de Oftalmologia da FMABC, operadas no período de julho de 2006 a junho de 2008 e com idade acima de 4 anos para obtenção de respostas verbais aos testes realizados.

\section{Métodos}

Foi realizado um estudo observacional com amostra não probabilística com todos os pacientes operados no período de julho de 2006 a junho de 2008.

Foram incluídos os pacientes com idade superior a 4 anos para obtenção de respostas verbais aos testes realizados, operadas por catarata diagnosticada na infância em ambos os olhos e implante de lente intra-ocular realizado em mesmo tempo cirúrgico. Foram excluídos os pacientes com idade inferior a 4 anos, pacientes com pós-operatório menor a 3 meses, portadores de patologias oculares associadas à catarata (como a da retinopatia da prematuridade ou associada à persistência do vítreo primário hiperplásico), cataratas secundárias a traumas ou uveítes, pacientes com doenças mentais, portadores de nistagmo e estrabismo prévio.

As variáveis coletadas foram sexo, idade atual, idade do aparecimento da catarata relatada pelo responsável, idade da cirurgia, acuidade visual pré-operatória, tempo de pós-operatório da última cirurgia, tempo de uso de oclusão pós- operatória, presença de opacidade de cápsula posterior e testes de função sensorial. As crianças avaliadas podem ou não ser portadoras de ametropias. As portadoras de ametropias foram testadas com a correção óptica em uso, prescrita após a cirurgia.em um escotoma foveal no olho $\mathrm{n}$ nas duas $\mathrm{f}$ outra, o oftalmologista ajusta um olho npara a vis

A função sensorial foi avaliada através de testes específicos, e foram realizados sucessivamente:

1) Acuidade visual (AV) (Tabela de Snellen): para longe e para perto, mono e binocular, com a correção óptica habitual. Consideramos a acuidade visual como boa maior ou igual a 0,4 .

2) Motilidade Ocular Extrínseca: detectar a presença ou não de estrabismo em um ou ambos os olhos.

3) Teste das 4 luzes de Worth: 4 luzes são exibidas ao paciente, à distância $(3 \mathrm{~m})$ e perto $(33 \mathrm{~cm})$, com o uso de lentes de filtro vermelho e verde (olho direito e esquerdo, respectivamente). A percepção de 2,3 ou 4 luzes indica se há ou não fusão periférica ou central. Consideramos a presença de fusão o reconhecimento das 4 luzes.

4) Teste estereoscópico de Titmus: Utilizam-se óculos polarizado e imagens vectográficas para investigar a acuidade estereoscópica através da percepção da profundidade das imagens. Consideramos estereopsia presente o resultado menor ou igual a 3000 segundos de 
arco. Menor ou igual a 400 segundos de arco foi considerada boa estereopsia.

A catarata foi considerada congênita quando relatado pelo familiar o aparecimento até o $3^{\circ}$ mês de vida; infantil precoce quando o aparecimento foi relatado após o terceiro mês de vida e infantil tardia quando o aparecimento após doze meses de vida.

A classificação quanto ao tipo de catarata variou quanto à apresentação da opacidade no cristalino, podendo ser: cortical (anterior e posterior), nuclear, subcapsular, nuclear embrionária, polar e pulverulenta.

A indicação cirúrgica das cataratas baseou-se no acometimento do eixo visual pela opacidade cristaliniana e na acuidade visual que a criança apresentava ao exame oftalmológico.

As variáveis correlacionadas foram idade do diagnóstico, Titmus e luzes de Worth; idade da primeira cirurgia e AV pós operatória; AV pré e pós operatória, Titmus e luzes de Worth.

Em todos os casos de opacidade de cápsula posterior foi realizado YAG laser logo após tempo de pósoperatório permitido (6 meses).

As variáveis estudadas foram submetidas a análise descritiva.

O nível de significância adotado neste estudo foi de $5 \%$.

\section{Resultados}

Foram avaliados cinqüenta prontuários de crianças com catarata infantil, operadas pelo setor de Oftalmopediatria da Faculdade de Medicina do ABC de julho de 2006 a junho de 2008, para seleção e convocação de pacientes que se adequavam aos critérios de inclusão deste estudo.

Foram selecionadas 12 (24\%) crianças, porém, 6 $(50 \%)$ não foram encontradas. Portanto, 6 crianças se adequavam aos critérios do trabalho. Cinco $(83,3 \%)$ eram do sexo feminino e 1 (16,7\%) do sexo masculino.

A média de idade das crianças foi de 10,50 anos $(\mathrm{DP}=3,391)$. A idade mínima foi de 5 anos e a máxima de 15 anos.

A média da idade do diagnóstico da catarata foi 5,58 anos $(\mathrm{DP}=3,35)$; apenas $1(16,7 \%)$ teve o diagnóstico aos 6 meses de idade.Todas as crianças já foram encaminhadas para este serviço com diagnóstico de catarata relatado pelo responsável.

A média da idade da primeira cirurgia foi de 8,83 $\operatorname{anos}(\mathrm{DP}=3,65)$.

Todas as crianças deste estudo realizaram extração da catarata pela técnica de facoemulsificação com colocação do implante intra-ocular no mesmo tempo de cirurgia. A média de tempo de pós-operatório mínimo foi de 10 meses ( $\mathrm{DP}=5,25)$.

Quanto ao tipo de catarata, 3 (50\%) eram nucleares e/ou corticais e/ou subcapsulares; 2 (33,3\%) eram pulverulentas e apenas $1(16,7 \%)$ era nuclear embrionária.

A acuidade visual (AV) pré-operatória média do olho direito foi de $0,21(\mathrm{DP}=0,11)$ e a do olho esquerdo foi $0,19(\mathrm{DP}=0,13)$. Não obtivemos acuidade visual préoperatória de um dos pacientes pois pela idade na época, não informava. A tabela 1 mostra as características clínicas pré-operatórias de cada paciente avaliado.

A média da acuidade visual (AV) pós-operatória do olho direito foi de $0,5(\mathrm{DP}=0,29)$; e, do olho esquerdo foi de $0,48(\mathrm{DP}=0,24)$. Três $(50 \%)$ dos olhos direitos e $4(67 \%)$ dos olhos esquerdos alcançaram AV pós-operatória melhor que 0,4 . Uma (16,7\%) criança apresentou como AV pós-operatória 1 e 0,8 (olho direito e esquerdo, respectivamente). Todas as AV pós-operatórias foram medidas com a correção atualizada.

Quanto aos testes de função sensorial binocular pós-operatório, apenas $1(16,7 \%)$ criança apresentou ausência da estereopsia ao teste de Titmus e não apresentou fusão no teste das luzes de Worth, tanto para perto quanto para longe. Essa criança apresentou padrão de supressão de olho esquerdo. Das 5(83\%) crianças que apresentaram boa função sensorial, todas apresentaram fusão no teste da luzes de Worth, tanto para perto quanto para longe. No teste de Titmus, 2 (33,3\%) apresentaram estereopsia grosseira, com 3000 segundos de arco e AV pós-operatória menor que 0,4 . As outras 3(50\%) apresentaram boa estereopsia com valor menor ou igual a 400 segundos de arco, com AV pós-operatória melhor que 0,4 . A tabela 2 mostra as características clínicas pósoperatórias de cada paciente avaliado.

A opacidade de cápsula posterior (OCP) apresentou-se como única complicação pós-operatória em 4 $(66,7 \%)$ dos pacientes, sendo realizado YAG laser, após 6 meses de pós-operatório. Nenhuma criança apresentou OCP no momento da aferição dos testes.

Nenhum dos pacientes apresentou alteração da motilidade extrínseca no período pós-operatório. Apenas $1(16,7 \%)$ criança do estudo está em uso de oclusão (quatro horas/dia) há 2 meses, na tentativa de melhora da ambliopia.

Houve diferença estatística $(\mathrm{p}<0,05)$ apenas entre AV pré e pós operatória de cada olho (Tabela 3 ).

A correlação entre a idade do diagnóstico com testes de Titmus e luzes de Worth não apresentaram significância estatística (OD: $\mathrm{p}=1,0 ; \mathrm{OE}$ : $\mathrm{p}=0,43$ ); assim 
Tabela 1

Características clínicas pré-operatórias

\begin{tabular}{cccccc}
\hline Paciente & $\begin{array}{c}\text { Idade } \\
\text { (anos) }\end{array}$ & $\begin{array}{c}\text { Classificação } \\
\text { da catarata }\end{array}$ & $\begin{array}{c}\text { Idade do } \\
\text { diagnóstico }\end{array}$ & AV pré operatória OD & AV pré operatória OE \\
\hline 1 & 10 & P 3-4+ & 8,0 & 0,17 & 0,13 \\
2 & 15 & NE & 5,0 & 0,20 & 0,13 \\
3 & 10 & N 2+/ CA e CP 2+ & 8,0 & 0,33 & 0,25 \\
4 & 5 & N+/CA e CP 1 & 3,0 & NÃO INFORMAVA & NÃO INFORMAVA \\
5 & 10 & P 3+ & 0,5 & 0,30 & 0,40 \\
6 & 13 & SCP 3+ & 9,0 & 0,05 & 0,05 \\
\hline
\end{tabular}

$\mathrm{p}=$ pulverulenta; $\mathrm{NE}=$ nuclear embrionária; $\mathrm{N}=$ nuclear; $\mathrm{CA}=$ cortical anterior; $\mathrm{CP}=$ cortical posterior; $\mathrm{SCP}=$ subcapsular. Classificação de + a $4+/ 4$.

Tabela 2

Características clínicas pós- operatórias

\begin{tabular}{|c|c|c|c|c|c|c|c|}
\hline Pacientes & $\begin{array}{l}\text { Idade (anos) } \\
\text { na } 1^{\mathrm{a}} \text { cirurgia }\end{array}$ & $\begin{array}{c}\text { Tempo de } \\
\text { pós operatório } \\
\text { mínimo (meses) } \\
\text { OD }\end{array}$ & $\begin{array}{c}\text { AV } \\
\text { pós operatória } \\
\text { OE }\end{array}$ & $\begin{array}{c}\text { AV } \\
\text { pós-operatória }\end{array}$ & $\begin{array}{c}\text { Titmus } \\
\text { (segundos de arco) }\end{array}$ & $\begin{array}{c}\text { Luzes de } \\
\text { Worth (PP) }\end{array}$ & $\begin{array}{c}\text { Luzes de } \\
\text { Worth (PL) }\end{array}$ \\
\hline 1 & 8 & 12 & 0,33 & 0,33 & 3000 & fusão & fusão \\
\hline 2 & 13 & 9 & 0,25 & 0,50 & 3000 & fusão & fusão \\
\hline 3 & 10 & 3 & 1,00 & 0,80 & 200 & fusão & fusão \\
\hline 4 & 3 & 6 & 0,25 & 0,10 & $\mathrm{O}$ & supressão & supressão \\
\hline 5 & 7 & 12 & 0,50 & 0,50 & 400 & fusão & fusão \\
\hline 6 & 12 & 18 & 0,67 & 0,67 & 200 & fusão & fusão \\
\hline
\end{tabular}

como a correlação entre idade da primeira cirurgia e AV pós operatória (OD: $\mathrm{p}=0,74 ; \mathrm{OE}: \mathrm{p}=0,2$ ). Também não foi observada correlação estatística entre AV préoperatória com Titmus (OD:p=0,8;OE: $\mathrm{p}=1,0)$ e AV pósoperatória com Titmus e luzes de Worth (OD: $\mathrm{p}=0,67$; $\mathrm{p}=0,27 ; \mathrm{OE}, \mathrm{p}=0,93 ; \mathrm{p}=0,15)$.

\section{Discussão}

A maioria dos estudos sobre função visual em cataratas congênitas e da infância avaliam crianças cujas cirurgias foram realizadas nos primeiros meses de vida e que, após o acompanhamento de quatro ou mais anos, quando a criança já adquiriu capacidade de informação, foram aplicados os testes de acuidade visual pela tabela de Snellen e testes de função sensorial, como o Titmus e as Luzes de Worth.

Os pacientes deste estudo apresentaram idade superior a quatro anos para que pudéssemos obter as informações aos testes, e todos foram operados em idade tardia, seja por diagnóstico tardio ou conduta expectante previamente adotada. Esses pacientes chegaram ao departamento de Oftalmologia da Faculdade de Medicina
Tabela 3

Correlação entre acuidades visuais pré e pós-operatórias entre os olhos

AV pré-operatória AV pós-operatória

OD

$\begin{array}{lcc}\text { MÉDIA } \pm \text { DP } & 0,21 \pm 0,11 & 0,5 \pm 0,29 \\ \text { Mín-Máx } & 0,05-0,33 & 0,25-1,00\end{array}$

OE

MÉDIA \pm DP $\quad 0,19 \pm 0,13 \quad 0,48 \pm 0,24$

Mín-Máx $\quad 0,05-0,4 \quad 0,10-0,80$

Wilcoxon Signed Ranks Test

do ABC já com o diagnóstico relatado pelo familiar; sendo neste serviço então indicadas e realizadas as cirurgias (após a avaliação oftalmológica completa).

A média de idade do diagnóstico da catarata dos pacientes avaliados $(5,58$ anos, $\mathrm{DP}=3,35)$ aponta para uma possível classificação (quanto à idade de aparecimento) como infantil tardia, ou como congênita ou infantil precoce, porém com desenvolvimento da opacidade lento.

Esses dados não puderam ser averiguados com 
precisão pois foram coletados a partir de informações dadas pelos responsáveis pelas crianças. Cinco (83\%) deles não relataram avaliação médica prévia ao período e apenas 1 (16,7\%) criança apresentou idade do diagnóstico aos seis meses de idade, porém, segundo informação coletada, foi adotada conduta expectante.

Cinquenta e $67 \%$ das crianças apresentaram acuidade visual pós-operatória melhor que 0,4 de olho direito e esquerdo respectivamente. Esses dados são compatíveis com um estudo brasileiro em que $48 \%$ das crianças pseudofácicas por catarata bilateral operadas entre 4 e 10 anos de idade, obtiveram boa acuidade visual ${ }^{9}$. Este resultado também é compatível com um estudo envolvendo 22 pacientes, em que 46,8\% apresentaram boa acuidade visual pós-operatória ${ }^{3}$. Outros estudos obtiveram os melhores resultados na acuidade visual pós-operatória de crianças com idade mais avançada tanto para diagnóstico quanto para cirurgia ${ }^{7,10}$. Esses estudos porém não relatam os dados da acuidade visual pré-operatória das crianças avaliadas.

Neste estudo o índice de função sensorial obtido foi satisfatório. Apenas uma (16,7\%) criança não apresentou estereopsia ao teste de Titmus e fusão nos testes de luzes de Worth. As outras 5(83\%) crianças apresentaram estereopsia e fusão nos testes. Duas (33\%) apresentaram estereopsia grosseira (3000 segundos de arco) e $3(50 \%)$ boa (menor ou igual a 400 segundos de arco); sendo que $2(33,3 \%)$ destas apresentaram 200 segundos de arco. Este dado difere da maior parte da literatura, em que a binocularidade foi atingida por menor porcentagem de pacientes e seus resultados ainda são considerados limitados; porém é compatível com dados de estudo semelhante com crianças operadas por catarata unilateral $3,10-12$.

Estudos mostram que as seis primeiras semanas de vida representam um período sensível para o desenvolvimento da binocularidade, e, que o grau de privação visual que ocorre pela catarata nesse período, determina variações em seu prognóstico. Outros, relatam que esse período ocorre do terceiro ao sexto mês do período pós-natal ${ }^{10,13}$.

Um outro fator determinante à binocularidade é a acuidade visual. Estudos antigos apontam que quando a AV binocular varia entre 20/200 e 20/20, a estereopsia muda proporcionalmente ${ }^{14}$. Zubcov et al apontam que a preservação da estereopsia precisa de pelo menos AV de 20/100 $(0,20)$ do pior olho ${ }^{6}$.Apenas $1(16,7 \%)$ criança deste estudo não obteve visão melhor que 0,20 ; sendo esta a única criança a não apresentar estereopsia ao teste de Titmus, nem fusão às luzes de Worth.

As cataratas bilaterais têm melhor prognóstico visual em relação às cataratas unilaterais ${ }^{3,4,10}$. Todos os pacientes avaliados apresentavam catarata bilateral, a maioria (50\%) dos casos corticais e/ou nucleares e/ ou subcapsulares, seguida pela pulverulenta(33,3\%). Esses dados diferem na literatura pelo fato da localização da opacidade (classificação da catarata) variar entre os estudos. Um estudo aponta 27,3\% de catarata nuclear e $40 \%$ de catarata subcapsular posterior ${ }^{3}$.

Nenhuma criança avaliada apresentou catarata total ou polar posterior, tidos como os tipos de catarata que mais causam ambliopia pela literatura ${ }^{4}$.

Assim, os bons índices de função sensorial binocular dos pacientes deste estudo podem ser atribuídos a três fatores: a não serem incluídos pacientes que apresentavam desvio ocular prévio ou nistagmo, a todas as cataratas serem bilaterais e à apresentação da opacidade limitante à visão ter sido em idade mais tardia, não interferindo no período de aprendizagem da binocularidade.

No entanto, obtivemos diferença estatística significativa apenas entre as acuidades visuais pré e pós operatórias. As outras variáveis que correlacionamos não apresentaram significância estatística, provavelmente devido à amostra reduzida, dado que na literatura, o resultado visual de olhos operados dependem da idade de início da catarata, idade de início da densidade da opacidade e idade da realização da cirurgia ${ }^{4,10}$. Outros estudos apontam significância estatística entre AV e função binocular, entre AV e idade da cirurgia ${ }^{3,10}$.

Houve neste estudo, alta incidência de opacidade de cápsula posterior pós-operatória (67\%). Um estudo mostrou $31,5 \%$ de opacidade de cápsula posterior, porém, essas crianças foram submetidas à lensectomia e implante de lente intraocular sem vitrectomia anterior, enquanto nossos pacientes foram submetidos à facoemulsificação com implante de lente intraocular ${ }^{10}$.

Outro estudo ( 22 olhos) obteve $56 \%$ de opacidade de cápsula posterior, porém a técnica cirúrgica adotada variava devido à abordagem de três tipos de catarata (congênita, do desenvolvimento e traumática) ${ }^{6}$.

\section{Conclusõ̃es}

Neste estudo, crianças portadoras de catarata congênita ou infantil precoce de desenvolvimento lento e de catarata infantil tardia, apresentaram bom prognóstico visual. A maioria apresentou função binocular após cirurgia com implante de lente intraocular, mesmo em idade avançada para cirurgia. Houve diferença estatíticamente significante entre as acuidades visuais 
pré e pós- operatórias de cada um dos olhos, entretanto, novos estudos devem ser realizados para melhor análise dos resultados.

\section{AbStRACT}

Objective: The privation ambliopia is the worst type of all. Among these, the most common cause is congenital cataract. The purpose of this study is to evaluate the visual function of children who had surgery between July 2006 and June 2008 for pediatric cataract. Methods: All children were above 4 years old and had surgery at advanced age.We evaluated 6 children and submitted them to visual acuity, extrinsic ocular movement, Titmus and Worth lights tests. Results: The mean age at the time of diagnosis was 5,58 years old and at the first surgery was 8,83 years old. Fifty and $67 \%$ of children achieved post operative visual acuity above 0,4 in right and left eyes, respectively. Just one $(16,7 \%)$ child did not achieve stereopsy and fusion on the sensorial function tests. The others presented fusion on Worth light test. At Titmus test, 2(33,3\%) showed gross stereopsy and $3(50 \%)$ good. There was statistically significant difference between pre and post visual acuity. Conclusion: Therefore, in this study, children with congenital or pediatric cataract whith late development showed a good visual prognosis after surgery using intraocular implans lens, even at advanced age.

Keywords: Cataract/congenital; Depth perception; Visual acuity

\section{ReferÊNCIAS}

1. Bicas HEA. Fisiologia da visão binocular. Arq Bras Oftalmol. 2004; 67(1):172-80.

2. Kanski JJ. Oftalmologia Clínica. Rio de Janeiro: Elsevier; 2004.p.536-8.

3. Park JH, Yu YS, Kim SJ, Choung HK. Visual function after primary posterior chamber intraocular lens implantation in pediatric unilateral cataract: stereopsis and visual acuity. Korean J Ophtalmol . 2007; 21(4): 195-200.

4. Maia NCF, Hofling ALL, Baikoff G. Avaliação da eficácia da facectomia com implante de lente intra-ocular na infância. Arq Bras Oftalmol. 2005; 68(6):735-41.

5. Moreno GL, Tartarella MB, Leal FAM. Refraction study on pseudophakic children. Arq Bras Oftalmol. 2005;68(3): 373-5.

6. Zubcov AA, Stahl E, Rossillion B, Nutzenberger A, Kohnen T, Ohrloff C, Stark N. Stereopsis after primary in the bag posterior chamber implantation in children. J AAPOS. 1999; 3(4):227-33.

7. Travi GM, Schnall BM, Lehman SS, Kelly CJ, Hug D, Hirakata VN, Calhoun JH. Visual outcome and success of amblyopia treatment in unilateral small posterior lens opacities and lenticonus initially treated nonsurgically. J AAPOS. 2005;9(5):449-54.
8. Crouch ER Jr, Pressman SH, Crouch ER. Posterior chamber intraocular lenses: long-term results in pediatric cataract patients. J Pediatr Ophthalmol Strabismus. 1995;32(4):210-8.

9. Tartarella MB, Kawakami LT, Scarpi MJ, Hayashi S, Bonomo PPO. Aspectos cirúrgicos em catarata congênita. Arq Bras Oftalmol.1995;58(1):24- 8.

10. Yamamoto M, Dogru M, Nakamura M, Shirabe H, Tsukahara Y, Sekiya Y. Visual Function following congenital cataract surgery. Jpn J Ophtalmol. 1998; 42(5): 411- 6.

11. Brown SM, Archer S, Monte ADM. Stereopsis and Binocular Vision after surgery for Unilateral Infantile cataract. J AAPOS. 1999;3(2): 109-13.

12. Lundvall A, Kugelberg U. Outcome after treatment of congenital bilateral cataract. Acta Ophthalmol Scand. 2002; 80(6):593-7.

13. Archer SM, Sondhi N, Helveston EM. Strabismus in Infancy. Ophtalmology. 1989; 96(1):133-7.

14. Levy NS, Glick BA. Stereoscopic Perception and Snellen visual acuity. Am J Oplhtalmol. 1974;78(4):722-4.

\section{Endereço para correspondência \\ Patrícia da Rocha Pitta Ferraz \\ Rua Duque de Caxias, 180 apto 01 - Vila Boa Vista \\ CEP: 09041-380 - Santo André - SP \\ E-mail: prpferraz@hotmail.com}

